\title{
Determinantal Representations of Semihyperbolic Polynomials
}

\author{
GrEG KNESE
}

\begin{abstract}
We prove a generalization of the Hermitian version of the Helton-Vinnikov determinantal representation for hyperbolic polynomials to the class of semihyperbolic polynomials, a strictly larger class, as shown by an example. We also prove that certain hyperbolic polynomials affine in two out of four variables divide a determinantal polynomial. The proofs are based on work related to polynomials with no zeros on the bidisk and tridisk.
\end{abstract}

\section{Introduction}

A homogeneous polynomial $P \in \mathbb{R}\left[x_{0}, x_{1}, \ldots, x_{n}\right]$ is hyperbolic of degree $d$ with respect to $e \in \mathbb{R}^{n+1}$ if $P(e) \neq 0$ and if for all $x \in \mathbb{R}^{n+1}$ the one-variable polynomial $t \mapsto P(x-t e)$ has only real zeros. This concept was originally studied by Gårding for its relation to PDE (see [7; 15]), but it—and the related concept of stable polynomials - has since become important to convex optimization, combinatorics, probability, and analysis. See the papers and surveys $[31 ; 14 ; 13 ; 34 ; 29$; $15 ; 26]$.

A deep result in the area is a determinantal representation for trivariate hyperbolic polynomials due to Helton and Vinnikov [16; 32], who solved a 1958 conjecture of Lax [17] (see [18]) and, as is mentioned in [15], can be used to develop the full Gårding theory of hyperbolicity.

THEOREM A. Let $p \in \mathbb{R}\left[x_{0}, x_{1}, x_{2}\right]$ be hyperbolic of degree $d$ with respect to $e_{2}$ and monic in $x_{2}$. Then, there exist $d \times d$ real symmetric matrices $A_{0}$ and $A_{1}$ such that

$$
p\left(x_{0}, x_{1}, x_{2}\right)=\operatorname{det}\left(x_{0} A_{0}+x_{1} A_{1}+x_{2} I\right) .
$$

If we relax the problem to finding self-adjoint matrices instead of real symmetric matrices, then proofs more amenable to computations are possible (see $[11 ; 30$; 33]). The resulting theorem is just as useful for most purposes.

TheOREM $\mathrm{A}^{*}$. Let $p \in \mathbb{R}\left[x_{0}, x_{1}, x_{2}\right]$ be hyperbolic of degree $d$ with respect to $e_{2}$ and monic in $x_{2}$. Then, there exist $d \times d$ self-adjoint matrices $A_{0}$ and $A_{1}$ such that

$$
p\left(x_{0}, x_{1}, x_{2}\right)=\operatorname{det}\left(x_{0} A_{0}+x_{1} A_{1}+x_{2} I\right) .
$$

Received December 17, 2014. Revision received May 30, 2016.

This research was supported by NSF grant DMS-1363239. 
Our immediate goal is to prove a generalization of this result based on a result of Geronimo et al. [8] and an extension to four variables based on a result of Bickel and Knese [2], whereas our larger goal is to advertise the close connection between determinantal representations of hyperbolic polynomials and sums of squares decompositions for multivariable Schur stable polynomials. See $[12 ; 10$; $22 ; 23]$ for background on the latter topic.

Our main result establishes a determinantal representation with the assumption of hyperbolicity weakened. We shall call a homogeneous polynomial $P \in$ $\mathbb{R}\left[x_{0}, x_{1}, \ldots, x_{n}\right]$ a semihyperbolic polynomial with respect to the direction $e \in$ $\mathbb{R}^{n+1} \backslash\{0\}$ if for every $x \in \mathbb{R}^{n+1}$ the univariate polynomial $t \mapsto P(x-t e)$ is either identically zero or only has real roots. The key distinction between hyperbolic and semihyperbolic polynomials is that we do not assume that $P(e) \neq 0$. Some references actually confuse the two, whereas Renegar [31] is the only reference we have found that emphasizes the distinction. We elaborate on our motivations in Section 6 . We do need to allow for $t \mapsto P(x-t e)$ to be identically zero because, for instance, if $P(e)=0$ and $x=0$, then $P(-t e) \equiv 0$. We give an example of a semihyperbolic polynomial that is not hyperbolic in any direction in Section 3.

Here is our main theorem.

TheOREM 1. Let $p \in \mathbb{R}\left[x_{0}, x_{1}, x_{2}\right]$ of degree $d$ be semihyperbolic with respect to $e_{2}=(0,0,1)$. Then, there exist $d \times d$ self-adjoint matrices $A_{0}, A_{1}$, and $A_{2}$ with $A_{2}$ positive semidefinite and a constant $c \in \mathbb{R}$ such that

$$
p(x)=c \operatorname{det}\left(x_{0} A_{0}+x_{1} A_{1}+x_{2} A_{2}\right) .
$$

Assuming that $p$ has no factors depending on $x_{0}, x_{1}$ alone, the data can be chosen to additionally satisfy:

$$
\operatorname{rank} A_{1}=\operatorname{deg}_{1} p, \quad \operatorname{rank} A_{2}=\operatorname{deg}_{2} p,
$$

- $A_{1}=B_{+}-B_{-}$with $B_{ \pm}$both positive semidefinite where rank $B_{-}$equals the number of roots of $p(1, t, i)$ in the upper half plane and $\operatorname{rank} B_{+}+\operatorname{rank} B_{-}=$ rank $A_{1}$,

- and $B_{-}+B_{+}+A_{2}=I$.

See Section 2 for the proof. We can recover Theorem $A^{*}$ when $p\left(e_{2}\right) \neq 0$ since $p$ will then have degree $d$ in $x_{2}$ and then $A_{2}$ will be positive definite. We can then factor $A_{2}^{1 / 2}$ from the right and left of $\sum_{j=0}^{2} x_{j} A_{j}$ in order to get a determinantal representation of the form given in Theorem $\mathrm{A}^{*}$, namely with $A_{2}=I$.

There is nothing special about the vector $e_{2}$; a linear change of variables could be used to establish a determinantal representation for other semihyperbolic polynomials. The assumption of no factors depending on only $x_{0}, x_{1}$ is there to avoid certain annoyances that such trivial factors introduce. For instance, $p\left(x_{0}, x_{1}, x_{2}\right)=x_{1}$ is certainly semihyperbolic in the direction $e_{2}$, but then $A_{2}=A_{0}=0$, and the signature of the $1 \times 1$ matrix $A_{1}$ does not really provide any useful information. 
It follows that a trivariate semihyperbolic polynomial $p$ can be lifted to a fourvariable polynomial

$$
P\left(x_{0}, x_{1}, y_{1}, x_{2}\right)=c \operatorname{det}\left(x_{0} A_{0}+x_{1} B_{+}+y_{1} B_{-}+x_{2} A_{2}\right),
$$

which is hyperbolic in the direction $(0,1,1,1)$ and such that $P\left(x_{0}, x_{1},-x_{1}, x_{2}\right)=$ $p\left(x_{0}, x_{1}, x_{2}\right)$. So, we are projecting a hyperbolic polynomial (possessing a definite determinantal representation) of four variables to a set where it is not necessarily hyperbolic. It also follows that a trivariate semihyperbolic polynomial is a limit of hyperbolic polynomials. Indeed, writing $p$ as in Theorem 1 , define, for $\varepsilon>0$,

$$
p_{\varepsilon}(z)=c \operatorname{det}\left(x_{0} A_{0}+x_{1} A_{1}+x_{2}\left(A_{2}+\varepsilon I\right)\right) .
$$

Then, $p_{\varepsilon} \rightarrow p$ as $\varepsilon \searrow 0$. We do not know if semihyperbolic polynomials in more than three variables are the limits of hyperbolic polynomials.

Theorem 1 has a curious asymmetry in its treatment of $x_{0}$ and $x_{1}$. This is partly due to idiosyncrasies of our proof, but we also think there are some subtleties to resolve. To be specific, we could certainly break up $A_{0}$ into a difference of positive semidefinite matrices according to its signature, but we have been unable to connect the signature of the $A_{0}$ we construct with geometric properties of $p$. We have no reason to believe this cannot be done, especially because this issue does not arise in the hyperbolic case. Indeed, we can take $A_{2}=I$, and the number of zeros of $t \mapsto p\left(t x_{0}, t x_{1}, i\right)$ in $\mathbb{C}_{+}$equals the number of negative eigenvalues of $x_{0} A_{0}+x_{1} A_{1}$. Similarly, the number of zeros of $t \mapsto p\left(t x_{0}, t x_{1},-i\right)$ equals the number of positive eigenvalues of $x_{0} A_{0}+x_{1} A_{1}$. Thus, the signature of $x_{0} A_{0}+$ $x_{1} A_{1}$ can be derived from properties of $p$ in the hyperbolic case. Notice that we evaluate $p$ on the complex line $(0, t, i)$ to determine the signature of $A_{1}$, whereas in the theorem we evaluate on the line $(1, t, i)$, which actually seems less natural. The example in Section 3 shows this is actually necessary: using the line $(1, t, i)$, we get a correct count of the negative eigenvalues of $A_{1}$, whereas using the line $(0, t, i)$, we get an incorrect count. The details are recorded in Section 3 .

As a nice corollary, we can quickly recover the following variant of Theorem $A^{*}$. The original proof, while not difficult, requires transforming a real stable polynomial to a hyperbolic polynomial through a linear transformation. Our signature count of $A_{1}$ in Theorem 1 makes the proof go smoothly.

Corollary 1 (See Theorem 6.6 of [3]). If $p \in \mathbb{R}\left[x_{0}, x_{1}, x_{2}\right]$ is homogeneous of degree $d$ and hyperbolic with respect to all vectors in the cone $\left\{\left(0, v_{1}, v_{2}\right)\right.$ : $\left.v_{1}, v_{2}>0\right\}$, then there exist $d \times d$ self-adjoint matrices $A_{0}, A_{1}$, and $A_{2}$ and $a$ constant $c \in \mathbb{R}$ such that $A_{1}$ and $A_{2}$ are positive semidefinite, $A_{1}+A_{2}=I$, and

$$
p(x)=c \operatorname{det}\left(x_{0} A_{0}+x_{1} A_{1}+x_{2} A_{2}\right) .
$$

Since [3] uses Theorem A to prove this result, all of the matrices can be taken to be real, but our proof does not yield this. For $p$ as in the corollary, $p\left(1, x_{1}, x_{2}\right)$ is known as a real stable polynomial. This formula was used in the recent paper regarding the Kadison-Singer problem [26]. See Section 4 for the very short proof of the corollary. 
The key tool for the proof of Theorem 1 is a determinantal representation proven by Geronimo, Iliev, and Knese [8] for certain polynomials on the bidisk $\mathbb{D}^{2}=\mathbb{D} \times \mathbb{D}$ (here $\mathbb{D}$ is the open unit disk in the complex plane $\mathbb{C}$ ). Define $D(z)=z_{1} D_{1}+z_{2} D_{2}$ where the $D_{1}$ and $D_{2}$ are $(n+m) \times(n+m)$ matrices given by

$$
D_{1}=\left(\begin{array}{cc}
I_{n} & 0 \\
0 & 0
\end{array}\right), \quad D_{2}=\left(\begin{array}{cc}
0 & 0 \\
0 & I_{m}
\end{array}\right) .
$$

For $n=n_{1}+n_{2}$, define

$$
P_{+}=\left(\begin{array}{ccc}
I_{n_{1}} & 0 & 0 \\
0 & 0 & 0 \\
0 & 0 & 0
\end{array}\right), \quad P_{-}=\left(\begin{array}{ccc}
0 & 0 & 0 \\
0 & I_{n_{2}} & 0 \\
0 & 0 & 0
\end{array}\right),
$$

where the blocks correspond to the orthogonal decomposition $\mathbb{C}^{n+m}=\mathbb{C}^{n_{1}} \oplus$ $\mathbb{C}^{n_{2}} \oplus \mathbb{C}^{m}$. Let $\mathbb{E}=\{z \in \mathbb{C}:|z|>1\}$ and $\mathbb{T}=\{z \in \mathbb{C}:|z|=1\}$.

Theorem B. Suppose $p \in \mathbb{C}\left[z_{1}, z_{2}\right]$ has bidegree $(n, m)$, no zeros in $(\mathbb{T} \times \mathbb{D}) \cup$ $(\mathbb{T} \times \mathbb{E})$, and no factors depending on $z_{1}$ alone. Let $n_{2}$ be the number of zeros of $p\left(z_{1}, 0\right)$ in $\mathbb{D}$. Then, there exists an $(n+m) \times(n+m)$ unitary $U$ and a constant $c \in \mathbb{C}$ such that

$$
p\left(z_{1}, z_{2}\right)=c \operatorname{det}\left(\left(z_{1} P_{-}+P_{+}+D_{2}\right)-U\left(P_{-}+z_{1} P_{+}+z_{2} D_{2}\right)\right) .
$$

This is referred to as a determinantal representation for "generalized distinguished varieties" in [8] since it generalizes a determinantal representation for the "distinguished varieties" of Agler and McCarthy [1], which correspond to the case $n_{2}=0$. Polynomials defining distinguished varieties are essentially a Cayley transform of real stable polynomials, and distinguished varieties have their own motivation in terms of operator theory as shown in [1]. Theorem B is based on first proving a sums-of-squares decomposition for polynomials $p \in \mathbb{C}\left[z_{1}, z_{2}\right]$ with no zeros in $\mathbb{T} \times \mathbb{D}$ ("a face of the bidisk") and no factors in common with $\tilde{p}(z)=z_{1}^{n} z_{2}^{m} \overline{p\left(1 / \bar{z}_{1}, 1 / \bar{z}_{2}\right)}$. Namely,

$$
|p(z)|^{2}-|\tilde{p}(z)|^{2}=\left(1-\left|z_{1}\right|^{2}\right)\left(\left|E_{1}(z)\right|^{2}-\left|E_{2}(z)\right|^{2}\right)+\left(1-\left|z_{2}\right|^{2}\right)|F(z)|^{2},
$$

where $E_{1} \in \mathbb{C}^{n_{1}}[z], E_{2} \in \mathbb{C}^{n_{2}}[z], F \in \mathbb{C}^{m}[z]$, and $n=n_{1}+n_{2}$ where $n_{2}$ is the number of zeros of $p\left(z_{1}, 0\right)$ in $\mathbb{D}$. This formula generalizes a sums-of-squares formula of Cole and Wermer [5] related to Andô's inequality from operator theory (see also [9] and [20]). It would be interesting to characterize which polynomials possess such a sums-of-squares formula where $|F(z)|^{2}$ is also given by a difference of squares $\left|F_{1}(z)\right|^{2}-\left|F_{2}(z)\right|^{2}$, and-going further-it would be interesting to see what sort of determinantal representation for real homogeneous polynomials comes out of the corresponding development from Theorem B to Theorem 1 presented here.

Beyond trivariate polynomials, there are many results on the existence or nonexistence of determinantal representations. See $[33 ; 19 ; 27 ; 28 ; 4 ; 25]$ for recent results and convenient summaries of the state of the art. Vinnikov [33] conjectures that hyperbolic polynomials always divide a hyperbolic polynomial 
that has a determinantal representation but with additional requirements placed on the set where the determinantal polynomial is positive. Our next theorem offers a step in the right direction for this conjecture albeit in a special situation. A polynomial $p$ is affine with respect to a variable $x_{j}$ if it has degree one in that variable.

TheOREM 2. Let $p \in \mathbb{R}\left[x_{0}, x_{1}, x_{2}, x_{3}\right]$ be hyperbolic of degree $d$ with respect to the cone $\left\{\left(0, v_{1}, v_{2}, v_{3}\right): v_{1}, v_{2}, v_{3}>0\right\}$. Assume that $p$ is affine in $x_{2}$ and $x_{3}$ and of degree $n$ in $x_{1}$. Then, there exist $k \leq 2 n+4$ and $k \times k$ self-adjoint matrices $A_{0}$, $A_{1}, A_{2}, A_{3}$ such that $p$ divides

$$
\operatorname{det}\left(\sum_{j=0}^{3} x_{j} A_{j}\right)
$$

$A_{1}, A_{2}, A_{3}$ are positive semi-definite, and $A_{1}+A_{2}+A_{3}=I$.

See Section 5. Theorem 2 seems to be one of the few higher-dimensional situations where we get a determinantal representation from simple hypotheses. The recent article of Kummer [24] proves the interesting result that a hyperbolic polynomial in $n$ variables with no real singularities divides a determinantal polynomial. This article also obtains bounds on the sizes of the matrices involved under the assumption that some power of the polynomial has a determinantal representation. Theorem 2 requires no assumptions of smoothness and obtains general bounds on the sizes of the matrices involved, but Kummer's result has the advantage that it works in $n$ variables and does not assume degree restrictions.

The key tool for Theorem 2 is the following sums-of-squares decomposition from Bickel and Knese [2].

TheOREM C (Theorem 1.12 of [2]). Let $p \in \mathbb{C}\left[z_{1}, z_{2}, z_{3}\right]$ have multidegree $(n, 1,1)$ and no zeros on $\overline{\mathbb{D}}^{3}$. Then, there exist column-vector-valued polynomials $E_{1} \in \mathbb{C}^{n}\left[z_{1}, z_{2}, z_{3}\right], E_{2}, E_{3} \in \mathbb{C}^{2}\left[z_{1}, z_{2}, z_{3}\right]$ such that, for $z=\left(z_{1}, z_{2}, z_{3}\right)$ and $w=\left(w_{1}, w_{2}, w_{3}\right)$,

$$
p(z) \overline{p(w)}-\tilde{p}(z) \overline{\tilde{p}(w)}=\sum_{j=1}^{3}\left(1-z_{j} \bar{w}_{j}\right) E_{j}(w)^{*} E_{j}(z)
$$

where $\tilde{p}(z)=z_{1}^{n} z_{2} z_{3} \overline{p\left(1 / \bar{z}_{1}, 1 / \bar{z}_{2}, 1 / \bar{z}_{3}\right)}$.

\section{Proof of Theorem 1 from Theorem $B$}

Let $\mathbb{C}_{+}=\{z \in \mathbb{C}: \Im z>0\}$ and $\mathbb{C}_{-}=\{z \in \mathbb{C}: \Im z<0\}$. $\mathbb{R}^{3}$,

Assume that $P \in \mathbb{R}\left[x_{0}, x_{1}, x_{2}\right]$ is homogeneous of degree $d$ and, for every $x \in$

$$
t \mapsto P\left(x-t e_{2}\right)
$$


is either identically zero or only has real zeros. We will assume that $P$ has no factors depending only on $x_{0}, x_{1}$, which can easily be incorporated into our final determinantal representation by appending diagonal blocks to our matrices.

Consider

$$
q\left(z_{1}, z_{2}\right)=P\left(1, z_{1}, z_{2}\right)
$$

which has no zeros in $\left(\mathbb{R} \times \mathbb{C}_{+}\right) \cup\left(\mathbb{R} \times \mathbb{C}_{-}\right)$. To see this, take $z=\left(a_{1}, a_{2}+i b_{2}\right) \in$ $\left(\mathbb{R} \times \mathbb{C}_{+}\right) \cup\left(\mathbb{R} \times \mathbb{C}_{-}\right)$with $q(z)=0$. Then, $P\left(\left(1, a_{1}, a_{2}\right)+t e_{2}\right)$ has the imaginary root $t=i b_{2}$, which would imply that $t \mapsto P\left(1, a_{1}, a_{2}+t\right)$ is identically zero. This means that $x_{1}-a_{1} x_{0}$ divides $P$, which we have ruled out.

Now, define

$$
p\left(z_{1}, z_{2}\right)=q\left(i \frac{1+z_{1}}{1-z_{1}}, i \frac{1+z_{2}}{1-z_{2}}\right)\left(\frac{1-z_{1}}{2 i}\right)^{n}\left(\frac{1-z_{2}}{2 i}\right)^{m}
$$

where $q$ has degree $n$ in $x_{1}$ and degree $m$ in $x_{2}$. Setting $x_{0}=1$ in $P\left(x_{0}, x_{1}, x_{2}\right)$ cannot lower the degree in $x_{1}$ or $x_{2}$, so $n=\operatorname{deg}_{1} P$ and $m=\operatorname{deg}_{2} P$. Recall that

$$
z \mapsto i \frac{1+z}{1-z}
$$

is a conformal map of the unit disk onto the upper half-plane sending $\mathbb{T}$ to $\mathbb{R} \cup\{\infty\}$ where $1 \mapsto \infty$. Thus, $p$ has no zeros in $(\mathbb{T} \backslash\{1\}) \times \mathbb{D}$ as well as $(\mathbb{T} \backslash\{1\}) \times \mathbb{E}$ where $\mathbb{E}=\{z \in \mathbb{C}:|z|>1\}$. We cannot have $p\left(1, z_{2}\right)=0$ unless $p\left(z_{1}, z_{2}\right)$ has $z_{1}-1$ as a factor. This follows by Hurwitz's theorem since the polynomials $z_{2} \mapsto$ $p\left(z_{1}, z_{2}\right)$ will have no zeros in $\mathbb{C} \backslash \mathbb{T}$ for $z_{1} \in \mathbb{T}$ with $z_{1} \rightarrow 1$, and then $p\left(1, z_{2}\right)$ will either have the same property or will be identically zero. However, such factors cannot exist since they imply that $q$ has degree less than $n$ in $x_{1}$. In any case, we can safely divide out factors of $p$ that depend only on $z_{1}$ since these can easily be incorporated into our final determinantal representation. Having done this, $p$ satisfies the hypotheses of Theorem B, and we may write

$$
p\left(z_{1}, z_{2}\right)=c \operatorname{det}\left(\left(z_{1} P_{-}+P_{+}+D_{2}\right)-U\left(P_{-}+z_{1} P_{+}+z_{2} D_{2}\right)\right)
$$

for a unitary $U$. Notice that $n_{2}$ is the number of roots of $z_{1} \mapsto p\left(z_{1}, 0\right)$ in $\mathbb{D}$, which is the same as the number of roots of $z_{1} \mapsto q\left(z_{1}, i\right)=P\left(1, z_{1}, i\right)$ in $\mathbb{C}_{+}$.

We convert back to $q$ via $z \mapsto \frac{z-i}{z+i}$. So,

$$
\begin{aligned}
q\left(z_{1}, z_{2}\right)= & p\left(\frac{z_{1}-i}{z_{1}+i}, \frac{z_{2}-i}{z_{2}+i}\right)\left(z_{1}+i\right)^{n}\left(z_{2}+i\right)^{m} \\
= & p\left(\frac{z_{1}-i}{z_{1}+i}, \frac{z_{2}-i}{z_{2}+i}\right) \operatorname{det}\left(\left(z_{1}+i\right) D_{1}+\left(z_{2}+i\right) D_{2}\right) \\
= & c \operatorname{det}\left(\left(z_{1}-i\right) P_{-}+\left(z_{1}+i\right) P_{+}+\left(z_{2}+i\right) D_{2}\right. \\
& \left.-U\left(\left(z_{1}+i\right) P_{-}+\left(z_{1}-i\right) P_{+}+\left(z_{2}-i\right) D_{2}\right)\right) \\
= & c \operatorname{det}\left((I-U) D(z)-i(I+U)\left(P_{-}-P_{+}-D_{2}\right)\right) \\
= & \pm c \operatorname{det}\left((I-U)\left(-z_{1} P_{-}+z_{1} P_{+}+z_{2} D_{2}\right)+i(I+U)\right) .
\end{aligned}
$$

The last line comes from multiplying on the right by $\operatorname{det}\left(-P_{-}+P_{+}+D_{2}\right)$. Letting $M(z)=-z_{1} P_{-}+z_{1} P_{+}+z_{2} D_{2}$, we now form the spectral decomposition $U=$ $V\left(\begin{array}{ll}u & 0 \\ 0 & I\end{array}\right) V^{*} ; V$ is a unitary, $u$ is a $k \times k$ diagonal unitary with no 1 s on the diagonal, 
and $k$ is the rank of $U-I$. Factoring $V$ and $V^{*}$ out from the left and right of (2.1) leaves

$$
\begin{aligned}
q(z) & = \pm c \operatorname{det}\left(\left(\begin{array}{cc}
I-u & 0 \\
0 & 0
\end{array}\right) V^{*} M(z) V+i\left(\begin{array}{cc}
I+u & 0 \\
0 & 2 I
\end{array}\right)\right) \\
& = \pm c \operatorname{det}(I-u) \operatorname{det}\left(\left(\begin{array}{ll}
I & 0 \\
0 & 0
\end{array}\right) V^{*} M(z) V+\left(\begin{array}{cc}
a & 0 \\
0 & 2 i I
\end{array}\right)\right) \\
& = \pm c \operatorname{det}(I-u) \operatorname{det}\left(\begin{array}{cc}
\left(V^{*} M(z) V\right)_{k k}+a & * \\
0 & 2 i I
\end{array}\right) \\
& =C \operatorname{det}\left(\left(V^{*} M(z) V\right)_{k k}+a\right)
\end{aligned}
$$

where $a=i(I+u)(I-u)^{-1}$ is a diagonal matrix with real entries, $\left(V^{*} M(z) V\right)_{k k}$ is the upper $k \times k$ block of $V^{*} M(z) M$, and $C$ is a constant. Now, $V^{*} M(z) V=$ $-z_{1} V^{*} P_{-} V+z_{1} V^{*} P_{+} V+z_{2} V^{*} D_{2} V$, and if we set $A_{0}=a, A_{1}=\left(-V^{*} P_{-} V+\right.$ $\left.V^{*} P_{+} V\right)_{k k}$, and $A_{2}=\left(V^{*} D_{2} V\right)_{k k}$, then we have a determinantal representation for $q$ :

$$
q(z)=C \operatorname{det}\left(A_{0}+z_{1} A_{1}+z_{2} A_{2}\right) .
$$

Notice that $A_{0}, A_{1}, A_{2}$ are evidently self-adjoint with $A_{2}$ positive semidefinite, and since $\operatorname{deg} q=d$, we have $d \leq k$. Once we show that $k=d$, we can homogenize to get the determinantal representation for $P$. It helps to first establish some of the additional details listed in Theorem 1.

It is a general fact that for matrices $A, B$, the $\operatorname{degree}$ of $\operatorname{det}(t A+B)$ is at most $\operatorname{rank} A$ (we leave this as an exercise). So, $\operatorname{deg}_{j} q \leq \operatorname{rank} A_{j}$ for $j=1,2$. On the other hand, by construction $\operatorname{rank} A_{1} \leq \operatorname{rank}\left(-P_{-}+P_{+}\right)=\operatorname{deg}_{1} q$ and $\operatorname{rank} A_{2} \leq \operatorname{rank} D_{2}=\operatorname{deg}_{2} q$, yielding $\operatorname{deg}_{j} q=\operatorname{rank} A_{j}$ for $j=1,2$. Next, setting $B_{ \pm}=\left(V^{*} P_{ \pm} V\right)_{k k}$, we have $A_{1}=B_{+}-B_{-}$. Since rank $A_{1}=n_{1}+n_{2}$, rank $B_{+} \leq$ $n_{1}$, and rank $B_{-} \leq n_{2}$, we must have equality in both inequalities. This also shows that the ranges of $B_{+}, B_{-}$have trivial intersection by considering dimensions. Since $P_{+}+P_{-}+D_{2}=I$, we must have $B_{+}+B_{-}+A_{2}=I$.

In order to show $k=d$, it suffices to show that $Q(t):=t A_{1}+A_{2}$ is nonsingular for some $t$. For then, there would be a $t_{0}$ such that

$$
t \mapsto q\left(t\left(t_{0}, 1\right)\right)
$$

has degree $k$, and since $q$ has degree $d$, we would have $k \leq d$ and thus $k=d$.

Note that $Q(t)=I+(t-1) B_{+}-(t+1) B_{-}$. By the spectral theorem

$$
B_{+}=\sum_{j=1}^{n_{1}} v_{j} v_{j} v_{j}^{*} \quad \text { and } \quad B_{-}=\sum_{j=1}^{n_{2}} \mu_{j} w_{j} w_{j}^{*}
$$

where $V=\left\{v_{1}, \ldots, v_{n_{1}}\right\}$ and $W=\left\{w_{1}, \ldots, w_{n_{2}}\right\}$ form orthonormal sets of eigenvectors corresponding to the positive eigenvalues $\left\{v_{1}, \ldots, v_{n_{1}}\right\}$ and $\left\{\mu_{1}, \ldots, \mu_{n_{2}}\right\}$ of $B_{+}$and $B_{-}$, respectively. Let $Y=\left\{y_{1}, \ldots, y_{k-n}\right\}$ be an orthonormal basis for the complement of $V$ and $W$. Then, $\mathcal{B}=V \cup W \cup Y$ is a basis for $\mathbb{C}^{k}$. Let $\mathcal{C}$ be a basis dual to $\mathcal{B}$. (Two bases $\left\{b_{1}, \ldots, b_{N}\right\}$ and $\left\{c_{1}, \ldots, c_{N}\right\}$ are dual if $b_{j}^{*} c_{k}=\delta_{j k}$.) 
The matrix for $Q(t)$ obtained by using $\mathcal{C}$ as a basis for the domain and $\mathcal{B}$ for the range is of the form

$$
\left(\begin{array}{ccc}
I+(t-1) d_{+} & 0 & 0 \\
0 & I-(t+1) d_{-} & 0 \\
0 & 0 & I
\end{array}\right)
$$

for diagonal matrices $d_{+}, d_{-}$containing the eigenvalues $v_{1}, \ldots, v_{n_{1}}, \mu_{1}, \ldots, \mu_{n_{2}}$ on the diagonal. The determinant of this vanishes for only finitely many $t$, and so $Q\left(t_{0}\right)$ is certainly nonsingular for some $t_{0}$. Thus, $k=d$, and we homogenize $q$ at degree $d$ to see that

$$
P(x)=C \operatorname{det}\left(x_{0} A_{0}+x_{1} A_{1}+x_{2} A_{2}\right) .
$$

This concludes the proof of Theorem 1 .

\section{Example}

Renegar [31] has an example of a polynomial that is semihyperbolic but not hyperbolic in any direction (see Section 2 of that paper); however, we have constructed an example that is more illustrative for our purposes.

Let

$$
p\left(x_{0}, x_{1}, x_{2}\right)=2 x_{0}^{2} x_{1}-\left(x_{0}^{2}+3 x_{1}^{2}\right) x_{2} .
$$

Then, $t \mapsto p\left(x-t e_{2}\right)$ clearly has only real roots for $x \in \mathbb{R}^{3}$ since this one-variable polynomial has degree 1 and real coefficients. Let

$$
\begin{gathered}
A_{0}=\frac{i}{3}\left(\begin{array}{ccc}
0 & -3 & -\sqrt{3} \\
3 & 0 & \sqrt{3} \\
\sqrt{3} & -\sqrt{3} & 0
\end{array}\right), \\
A_{1}=\left(\begin{array}{ccc}
0 & 0 & 0 \\
0 & 1 & 0 \\
0 & 0 & -1
\end{array}\right), \quad A_{2}=\left(\begin{array}{lll}
1 & 0 & 0 \\
0 & 0 & 0 \\
0 & 0 & 0
\end{array}\right) .
\end{gathered}
$$

We see that

$$
p(x)=3 \operatorname{det}\left(x_{0} A_{0}+x_{1} A_{1}+x_{2} A_{2}\right) .
$$

As remarked in the Introduction, we can lift to

$$
P\left(x_{0}, x_{1}, y_{1}, x_{2}\right)=3 x_{1} y_{1} x_{2}-\left(x_{2}+x_{1}+3 y_{1}\right) x_{0}^{2},
$$

which is hyperbolic in the direction $(0,1,1,1)$ and such that $P\left(x_{0}, x_{1},-x_{1}, x_{2}\right)=$ $p\left(x_{0}, x_{1}, x_{2}\right)$. We now explain why $p$ is not hyperbolic in any direction.

We first show that $\{x: p(x) \neq 0\}$ consists of the two connected components $\mathcal{P}_{+}=\{x: p(x)>0\}$ and $\mathcal{P}_{-}=\{x: p(x)<0\}$. I thank the referee for the following simplified explanation. The hypersurface $\{x: p(x)=0\}$ is the graph of the continuous function $\left(x_{0}, x_{1}\right) \mapsto 2 x_{0}^{2} x_{1} /\left(x_{0}^{2}+3 x_{1}^{2}\right)$. Thus, $\{x: p(x) \neq 0\}$ is divided into exactly two components, the part above the graph and the part below.

Next, neither component $\mathcal{P}_{+}, \mathcal{P}_{-}$is convex. For instance, $(-1,0,-1),(1,0$, $-1) \in P_{+}$, but $(0,0,-1) \notin \mathcal{P}_{+}$. We can similarly show that $\mathcal{P}_{-}$is not convex. This implies that $p$ is not hyperbolic in any direction since it is a fundamental 
result of Gårding that if $p$ is hyperbolic in some direction $e$, then the connected component of $\{x: p(x) \neq 0\}$ containing $e$ is convex.

This brings up a potential paradox. Since $p$ is a limit of hyperbolic polynomials $p_{\varepsilon}$ as in equation (1.1), how is it possible that the connected components of $\{x$ : $p(x) \neq 0\}$ are nonconvex in our example? An answer is that a convex component of $\left\{x: p_{\varepsilon}(x) \neq 0\right\}$ could shrink to an isolated point (in projective space) as $\varepsilon \searrow 0$. This is something we have seen graphically using our example.

Finally, in connection with our discussion after Theorem 1 regarding the signatures of $A_{0}$ and $A_{1}$, let us point out that

$$
p(1, t, i)=2 t-\left(1+3 t^{2}\right) i
$$

has one zero in $\mathbb{C}_{+}$, which agrees with the number of negative eigenvalues of $A_{1}$. On the other hand, $p(0, t, i)=-3 t^{2} i$ has no zeros in $\mathbb{C}_{+}$. Notice also that $p(t, 1, i)=(2-i) t^{2}-3 i$ has one zero in $\mathbb{C}_{+}$. This matches the number of negative eigenvalues of $A_{0}$, which is what we would like to have more generally in order for Theorem 1 to have a more symmetric statement.

\section{Proof of Corollary 1}

Notice that $t \mapsto p\left(x-t e_{2}\right)$ is either identically zero or only has real roots by Hurwitz's theorem since this polynomial can be obtained as the limit as $a \searrow 0$ of

$$
t \mapsto p\left(x-t\left(a e_{1}+e_{2}\right)\right) .
$$

Any factors depending only on $x_{0}, x_{1}$ can easily be dealt with separately, so we may assume that there are no such factors. So, $p$ satisfies the hypotheses of Theorem 1. Also, $t \mapsto p(1, t, i)$ can have no zeros in the upper half-plane for if it had such a zero $z=x+i y$ where $y>0$, then

$$
t \mapsto p((1, x, 0)+t(0, y, 1))
$$

would have the nonreal zero $t=i$, contradicting hyperbolicity in the direction $(0, y, 1)$. This shows that $\operatorname{rank} B_{-}=0$ in Theorem 1 and therefore $A_{1}$ is positive semidefinite, as desired.

\section{Proof of Theorem 2 from Theorem}

We largely follow the scheme of [21]. Let $P \in \mathbb{R}\left[x_{0}, x_{1}, x_{2}, x_{3}\right]$ be homogeneous of degree $d$, of degree 1 in $x_{2}, x_{3}$, and of degree $n$ in $x_{1}$. Assume that $P$ is hyperbolic with respect to the cone $\left\{\left(0, v_{1}, v_{2}, v_{3}\right): v_{1}, v_{2}, v_{3}>0\right\}$. Then, for $x=\left(x_{1}, x_{2}, x_{3}\right)$,

$$
q(x)=P(1, x)
$$

has no zeros in $\mathbb{C}_{+}^{3} \cup \mathbb{C}_{-}^{3}$, and $\overline{q(\bar{x})}=q(x)$. Switching to the tridisk, we see that

$$
f(z)=q\left(i \frac{1+z_{1}}{1-z_{1}}, i \frac{1+z_{2}}{1-z_{2}}, i \frac{1+z_{3}}{1-z_{3}}\right)\left(\frac{1-z_{1}}{2 i}\right)^{n}\left(\frac{1-z_{2}}{2 i}\right)\left(\frac{1-z_{3}}{2 i}\right)
$$


has no zeros in $\mathbb{D}^{3} \cup \mathbb{E}^{3}$. Note that we may as well assume that $f$ is irreducible since otherwise $f$ would have a factor depending on one or two variables alone, in which case there is no issue with having a determinantal representation.

Let $1 / \bar{z}=\left(1 / \bar{z}_{1}, 1 / \bar{z}_{2}, 1 / \bar{z}_{3}\right)$ for $z \in \mathbb{C}^{3}$ and define

$$
\begin{aligned}
\tilde{f}(z) & =z_{1}^{n} z_{2} z_{3} \overline{f(1 / \bar{z})}, \\
\frac{\widetilde{\partial f}}{\partial z_{j}} & =\frac{z_{1}^{n} z_{2} z_{3}}{z_{j}} \overline{\frac{\partial f}{\partial z_{j}}(1 / \bar{z})} \quad \text { for } j=1,2,3 .
\end{aligned}
$$

Since $q$ has real coefficients, we can show that $\tilde{f}=f$ and

$$
\begin{aligned}
n f & =z_{1} \frac{\partial f}{\partial z_{1}}+\frac{\widetilde{\partial f}}{\partial z_{1}}, \\
f & =z_{j} \frac{\partial f}{\partial z_{j}}+\frac{\widetilde{\partial f}}{\partial z_{j}} \quad \text { for } j=2,3
\end{aligned}
$$

after some simple computations. Thus, $(n+2) f=p+\tilde{p}$ where

$$
p(z)=\sum_{j=1}^{3} \frac{\widetilde{\partial f}}{\partial z_{j}}, \quad \tilde{p}(z)=\sum_{j=1}^{3} z_{j} \frac{\partial f}{\partial z_{j}} .
$$

Let $f_{t}(z)=f(t z)$ for $0<t<1$. Then, $f_{t}$ has no zeros in $\overline{\mathbb{D}}^{3}$, and if we set $\tilde{f}_{t}(z)=$ $t^{n+2} f(z / t)$, then $\left|f_{t}\right|=\left|\tilde{f}_{t}\right|$ on $\mathbb{T}^{3}$ (since $\tilde{f}=f$ ), and so $\tilde{f}_{t} / f_{t}$ is analytic and bounded by 1 in modulus for $z \in \mathbb{D}^{3}$ by the maximum principle. Now, for $z \in \overline{\mathbb{D}}^{3}$,

$$
\begin{aligned}
0 & \leq \lim _{t \nearrow 1} \frac{|f(t z)|^{2}-\left|t^{n+2} f(z / t)\right|^{2}}{1-t^{2}}(n+2) \\
& =(n+2)^{2}|f(z)|^{2}-2 \operatorname{Re}(\tilde{p}(z)(n+2) \overline{f(z)}) \\
& =|p(z)|^{2}-|\tilde{p}(z)|^{2} \quad \text { since }(n+2) f=p+\tilde{p}
\end{aligned}
$$

with some computations omitted (see [21] for more details). This shows that if $p$ vanishes in $\mathbb{D}^{3}$, then so does $\tilde{p}$, and so does $f$, which by assumption does not happen. Hence, $p$ has no zeros in $\mathbb{D}^{3}$.

Note that if $p$ and $\tilde{p}$ had a common factor, then this would be a factor of $f$, which we have already ruled out; we point out that $p$ and $\tilde{p}$ cannot be multiples of one another since $\tilde{p}$ vanishes at the origin. The conclusion of Theorem $C$ holds for such a $p$, but since we have only stated it for polynomials with no zeros on $\overline{\mathbb{D}}^{3}$ (as opposed to $\mathbb{D}^{3}$ ), we must explain how to address the case at hand. The main point is that for $0<t<1, p_{t}(z)=p(t z)$ will satisfy the hypotheses of Theorem $\mathrm{C}$, and therefore there exist vector polynomials $E_{1}^{t}, E_{2}^{t}, E_{3}^{t}$ corresponding to $p_{t}$ as in Theorem C. Then,

$$
\sup _{\mathbb{T}^{3}}|p(z)|^{2} \geq\left(1-\left|z_{j}\right|^{2}\right)\left|E_{j}^{t}(z)\right|^{2}
$$

shows that the vector polynomials $E_{j}^{t}$ are locally bounded in $\mathbb{D}^{3}$, and hence we can choose subsequences of $t \nearrow 1$ such that $E_{1}^{t} \in \mathbb{C}^{2 n}[z], E_{2}^{t}, E_{3}^{t} \in \mathbb{C}^{2}[z]$ converge to vector polynomials $E_{1} \in \mathbb{C}^{2 n}[z], E_{2}, E_{3} \in \mathbb{C}^{2}[z]$, and hence we will get 
a sums-of-squares decomposition as in Theorem C. Note the polynomials in $E_{1}$, $E_{2}, E_{3}$ necessarily have degree at most $(n-1,1,1),(n, 0,1),(n, 1,0)$ (this is proven in [23] for instance), and they will be nontrivial since $p$ and $\tilde{p}$ have no factors in common. On the zero set $Z_{f}$ of $f, p=-\tilde{p}$, and therefore

$$
0=\sum_{j=1}^{3}\left(1-z_{j} \bar{w}_{j}\right) E_{j}(w)^{*} E_{j}(z)
$$

for $z, w \in Z_{f}$. This equation ensures that the map

$$
\left(\begin{array}{l}
z_{1} E_{1}(z) \\
z_{2} E_{2}(z) \\
z_{3} E_{3}(z)
\end{array}\right) \mapsto\left(\begin{array}{l}
E_{1}(z) \\
E_{2}(z) \\
E_{3}(z)
\end{array}\right)
$$

defined initially for vectors of the above form with $z \in Z_{f}$, extends linearly to a well-defined $(2 n+4) \times(2 n+4)$ unitary $U$. Some details: If a combination of vectors from the left side of (5.2) sums to zero, then (5.1) shows the corresponding combination on the right sums to zero. So, we get a well-defined linear map from the span of the left side of (5.2) to the span of the right side. Now, (5.1) shows this map is an isometry. Since we are in finite dimensions, it can be extended to a unitary.

Note that $E_{1}, E_{2}, E_{3}$ cannot vanish identically in $Z_{f}$ without vanishing in all of $\mathbb{C}^{3}$ since the degrees are lower and $f$ is irreducible. Let $P_{j}$ for $j=1,2,3$ be the projection onto the $j$ th component in the orthogonal decomposition of $\mathbb{C}^{2 n+4}=\mathbb{C}^{2 n} \oplus \mathbb{C}^{2} \oplus \mathbb{C}^{2}$, and let $M(z)=\sum_{j=1}^{3} z_{j} P_{j}$. By (5.2), for $z \in Z_{f}$,

$$
(I-U M(z))\left(\begin{array}{l}
E_{1}(z) \\
E_{2}(z) \\
E_{3}(z)
\end{array}\right)=0,
$$

and therefore $\operatorname{det}(I-U M(z))=0$ for $z \in Z_{f} \backslash\left\{z: E_{1}, E_{2}, E_{3}=0\right\}$. Basic results in algebraic geometry (such as in Chap. 4 , Sect. 4 of [6]) can be used to establish that this implies that $\operatorname{det}(I-U M(z))$ vanishes for $z \in Z_{f}$ (i.e., $Z_{f} \backslash\left\{z: E_{1}, E_{2}, E_{3}=0\right\}$ is Zariski dense in $Z_{f}$ ) since $f$ is irreducible and none of $E_{1}, E_{2}, E_{3}$ vanishes identically on $Z_{f}$.

Therefore, $f$ divides $\operatorname{det}(I-U M(z))$. Write

$$
f(z) g(z)=\operatorname{det}(I-U M(z))
$$

for some polynomial $g$ of degree at most $(n, 1,1)$. As with Section 2, we convert back to $q$. There is some repetition in what follows, but since the situations are slightly different, we include the details. Now,

$$
\begin{aligned}
q(z) r(z) & =\operatorname{det}\left(\left(\sum_{j=1}^{3}\left(z_{j}+i\right) P_{j}\right)-U\left(\sum_{j=1}^{3}\left(z_{j}-i\right) P_{j}\right)\right) \\
& =\operatorname{det}((I-U) M(z)+i(I+U))
\end{aligned}
$$


for

$$
r(z)=\left(z_{1}+i\right)^{n}\left(z_{2}+i\right)\left(z_{3}+i\right) g\left(\frac{z_{1}-i}{z_{1}+i}, \frac{z_{2}-i}{z_{2}+i}, \frac{z_{3}-i}{z_{3}+i}\right) .
$$

Let $U=V\left(\begin{array}{ll}u & 0 \\ 0 & I\end{array}\right) V^{*}$ be the spectral decomposition of $U$ where $u$ is $k \times k$ diagonal with unimodular entries, none of which equals 1 . Here $k$ is the rank of $I-U$. As in Section 2, the determinant (5.3) can be converted to

$$
q(z) r(z)=(\text { const }) \operatorname{det}\left(\left(V^{*} M(z) V\right)_{k k}+a\right)
$$

where again $\left(V^{*} M(z) V\right)_{k k}$ refers to taking the upper $k \times k$ block of the given matrix, and $a=i(I+u)(I-u)^{-1}$. Finally, if we homogenize (5.4) at degree $k$-note this is at most $2 n+4$-then

$$
P(x) R(x)=(\text { const }) \operatorname{det}\left(x_{0} a+\sum_{j=1}^{3} x_{j} A_{j}\right)
$$

with $A_{j}=\left(V^{*} P_{j} V\right)_{k k}$ and $A_{1}+A_{2}+A_{3}=\left(V^{*} I V\right)_{k k}=I$, and where $R(x)=$ $x_{0}^{k-d} r\left(\left(1 / x_{0}\right)\left(x_{1}, x_{2}, x_{3}\right)\right)$. This concludes the proof.

\section{Concluding Questions and Remarks}

We think it is worth discussing or rehashing some of the motivations and lingering questions of this paper in more detail.

Semihyperbolic polynomials have perhaps been overlooked because they lack one of the key features of hyperbolic polynomials. Specifically, if $p$ is hyperbolic in the direction $e$, then the connected component of $\{x: p(x) \neq 0\}$ containing $e$ is convex (see [31]). No such result holds for semihyperbolic polynomials (see Renegar [31], Section 2, or Section 3 of this paper). This convexity property ties hyperbolic polynomials to optimization and is "the cornerstone of hyperbolic programming" [31]. This begs the question why study semihyperbolic polynomials, which may lack this property?

First, we think it is a good general principle in mathematics to understand the degenerate versions of objects of interest. Notice that the (local uniform) limit of a sequence of homogeneous polynomials of degree $d$ that are semihyperbolic with respect to a specific direction $e$ is either semihyperbolic or identically zero. This follows from Hurwitz's theorem applied to each polynomial $t \mapsto P(x-$ te). Hyperbolic polynomials do not share this property. Somewhat related is the following question mentioned in the Introduction.

QUESTION 1. Every trivariate semihyperbolic polynomial is a limit of hyperbolic polynomials. Is this true in the case of more variables?

Second, our main theorem, Theorem 1, shows that trivariate semihyperbolic polynomials possess determinantal representations just as in the hyperbolic case. We think this in itself provides a good justification for the study of semihyperbolic polynomials. This is a good point to formally state a question from the Introduction. 
Question 2. In Theorem 1, can the signature and rank of $A_{0}$ be determined directly from properties of $p$ ?

Our own personal motivations for studying semihyperbolic polynomials came from the natural connection we presented between semihyperbolic polynomials and two-variable polynomials with no zeros on $\mathbb{T} \times \mathbb{D}$. These latter polynomials appeared in [8] essentially because of the realization that some of the theory of polynomials with no zeros in $\mathbb{D}^{2}$ could be pushed further to the situation of no zeros in $\mathbb{T} \times \mathbb{D}$. It was realized later that this initially unnatural condition is closely related to hyperbolicity and indeed is essentially equivalent to semihyperbolicity.

Finally, we wish to rehash our larger goal of the paper of connecting sums of squares formulas to determinantal representations. This description will be somewhat imprecise. The approach of this paper shows that if $p\left(z_{1}, z_{2}, \ldots, z_{n}\right)$ has no zeros in $\mathbb{D}^{n}$ and possesses a Hermitian sums-of-squares formula

$$
|p|^{2}-|\tilde{p}|^{2}=\sum_{j=1}^{n}\left(1-\left|z_{j}\right|^{2}\right) \operatorname{SOS}_{j}
$$

(here each $\operatorname{SOS}_{j}$ term is a sum of squared moduli of polynomials), then $p+\tilde{p}$ divides a unitary determinantal polynomial

$$
\operatorname{det}(I-U D(z))
$$

Here $U$ is a unitary matrix, and $D(z)$ is a diagonal matrix with coordinate functions on the diagonal. We can then convert $p+\tilde{p}$ via the Cayley transform and homogenization to a hyperbolic polynomial (hyperbolic with respect to all vectors with positive entries) and through some linear algebra get a self-adjoint determinantal polynomial. We can reverse engineer some of this: take a hyperbolic polynomial $P$ (again hyperbolic with respect to vectors with positive entries) and convert to a polynomial $q$ satisfying $q=\tilde{q}$. If $q$ can be written as $p+\tilde{p}$ where $p$ satisfies (6.1), then $P$ divides a determinantal representation. If $n>2$, not every $p \in \mathbb{C}\left[z_{1}, \ldots, n\right]$ with no zeros in $\mathbb{D}^{n}$ satisfies an equation of the form (6.1); such polynomials are called Agler denominators. A polynomial is an Agler denominator if and only if $\tilde{p} / p$ satisfies a multivariable von Neumann inequality (see [23]).

We have also presented a modification to hyperbolicity/semihyperbolicity with respect to a specific direction. In $n$ variables, this would entail, after various conversions, to understanding polynomials satisfying (6.1) where $S O S_{1}, \ldots, S O S_{n-1}$ are replaced with differences of squares. With the exception of our work in [8], this is relatively uncharted territory.

ACKNOWLEDGMENTS. I would like to sincerely thank my coauthors J. Geronimo and P. Iliev [8], my coauthor K. Bickel [2], and the anonymous referee. I would like to also thank Victor Vinnikov for introducing me to hyperbolic polynomials. 


\section{References}

[1] J. Agler and J. E. McCarthy, Distinguished varieties, Acta Math. 194 (2005), no. 2, 133-153, doi:doiurl10.1007/BF02393219.

[2] K. Bickel and G. Knese, Inner functions on the bidisk and associated Hilbert spaces, J. Funct. Anal. 265 (2013), no. 11, 2753-2790, doi:doiurl10.1016/j.jfa.2013.08.002.

[3] J. Borcea and P. Brändén, Multivariate Pólya-Schur classification problems in the Weyl algebra, Proc. Lond. Math. Soc. (3) 101 (2010), no. 1, 73-104, doi:10.1112/plms/pdp049.

[4] P. Brändén, Obstructions to determinantal representability, Adv. Math. 226 (2011), no. 2, 1202-1212, doi:10.1016/j.aim.2010.08.003.

[5] B. J. Cole and J. Wermer, Ando's theorem and sums of squares, Indiana Univ. Math. J. 48 (1999), no. 3, 767-791, doi:10.1512/iumj.1999.48.1716.

[6] D. Cox, J. Little, and D. O'Shea, Ideals, varieties, and algorithms: an introduction to computational algebraic geometry and commutative algebra, Undergrad. Texts Math., 3rd edition, Springer, New York, 2007.

[7] L. Gårding, An inequality for hyperbolic polynomials, J. Math. Mech. 8 (1959), 957965.

[8] J. S. Geronimo, P. Iliev, and G. Knese, Polynomials with no zeros on a face of the bidisk, J. Funct. Anal. 270 (2016), no. 9, 3505-3558, doi:10.1016/j.jfa.2016.02.002.

[9] J. S. Geronimo and H. J. Woerdeman, Positive extensions, Fejér-Riesz factorization and autoregressive filters in two variables, Ann. of Math. (2) 160 (2004), no. 3, 839906, doi:10.4007/annals.2004.160.839.

[10] A. Grinshpan, D. S. Kaliuzhnyi-Verbovetskyi, V. Vinnikov, and H. J. Woerdeman, Classes of tuples of commuting contractions satisfying the multivariable von Neumann inequality, J. Funct. Anal. 256 (2009), no. 9, 3035-3054, doi:10.1016/j.jfa.2008.09.012.

[11] Stable and real-zero polynomials in two variables, Multidimens. Syst. Signal Process. 27 (2016), no. 1, 1-26, doi:10.1007/s11045-014-0286-3.

[12] A. Grinshpan, D. S. Kaliuzhnyi-Verbovetskyi, and H. J. Woerdeman, Normconstrained determinantal representations of multivariable polynomials, Complex Anal. Oper. Theory 7 (2013), no. 3, 635-654, doi:10.1007/s11785-012-0262-6.

[13] L. Gurvits, Combinatorics hidden in hyperbolic polynomials and related topics, 2004, arXiv:math/0402088v1.

[14] _ Van der Waerden/Schrijver-Valiant like conjectures and stable (aka hyperbolic) homogeneous polynomials: one theorem for all, Electron. J. Combin. 15 (2008), no. 1, Research Paper 66, 26. With a corrigendum.

[15] F. R. Harvey and H. B. Lawson Jr., Gårding's theory of hyperbolic polynomials, Comm. Pure Appl. Math. 66 (2013), no. 7, 1102-1128.

[16] J. W. Helton and V. Vinnikov, Linear matrix inequality representation of sets, Comm. Pure Appl. Math. 60 (2007), no. 5, 654-674, doi:10.1002/cpa.20155.

[17] P. D. Lax, Differential equations, difference equations and matrix theory, Comm. Pure Appl. Math. 11 (1958), 175-194.

[18] A. S. Lewis, P. A. Parrilo, and M. V. Ramana, The Lax conjecture is true, Proc. Amer. Math. Soc. 133 (2005), no. 9, 2495-2499 (electronic), doi:doiurl10.1090/S00029939-05-07752-X.

[19] D. Kerner and V. Vinnikov, Determinantal representations of singular hypersurfaces in $\mathbb{P}^{n}$, Adv. Math. 231 (2012), no. 3-4, 1619-1654, doi:10.1016/j.aim.2012.06.014.

[20] _ Polynomials with no zeros on the bidisk, Anal. PDE 3 (2010), no. 2, 109149, doi:10.2140/apde.2010.3.109. 
[21] G. Knese, Polynomials defining distinguished varieties, Trans. Amer. Math. Soc. 362 (2010), no. 11, 5635-5655, doi:10.2140/apde.2010.3.109.

[22] Schur-Agler class rational inner functions on the tridisk, Proc. Amer. Math. Soc. 139 (2011), no. 11, 4063-4072, doi:10.1090/S0002-9939-2011-10975-4.

[23] _ Rational inner functions in the Schur-Agler class of the polydisk, Publ. Mat. 55 (2011), no. 2, 343-357, doi:10.1090/S0002-9939-2011-10975-4.

[24] M. Kummer, Determinantal representations and Bézoutions, Math. Z. (2016), doi:10.1007/s00209-016-1715-9.

[25] M. Kummer, D. Plaumann, and C. Vinzant, Hyperbolic polynomials, interlacers, and sums of squares, Math. Program., Ser. B 153 (2015), no. 1, 223-245, doi:10.1007/s10107-013-0736-y.

[26] A. W. Marcus, D. A. Spielman, and N. Srivastava, Interlacing families II: mixed characteristic polynomials and the Kadison-Singer problem, Ann. of Math. (2) 182 (2015), no. 1, 327-350, doi:10.4007/annals.2015.182.1.8.

[27] T. Netzer and A. Thom, Polynomials with and without determinantal representations, Linear Algebra Appl. 437 (2012), no. 7, 1579-1595, doi:10.1016/j.laa.2012.04.043.

[28] T. Netzer, D. Plaumann, and A. Thom, Determinantal representations and the Hermite matrix, Michigan Math. J. 62 (2013), no. 2, 407-420, doi:10.1307/mmj/1370870379.

[29] R. Pemantle, Hyperbolicity and stable polynomials in combinatorics and probability, Current developments in mathematics, 2011, pp. 57-123, Int. Press, Somerville, MA, 2012.

[30] D. Plaumann and C. Vinzant, Determinantal representations of hyperbolic plane curves: an elementary approach, J. Symbolic Comput. 57 (2013), 48-60, doi:10.1016/j.jsc.2013.05.004.

[31] J. Renegar, Hyperbolic programs, and their derivative relaxations, Found. Comput. Math. 6 (2006), no. 1, 59-79, doi:10.1007/s10208-004-0136-z.

[32] V. Vinnikov, Selfadjoint determinantal representations of real plane curves, Math. Ann. 296 (1993), no. 3, 453-479, doi:10.1007/BF01445115.

[33] _ LMI representations of convex semialgebraic sets and determinantal representations of algebraic hypersurfaces: past, present, and future, Mathematical methods in systems, optimization, and control, Oper. Theory Adv. Appl., 222, pp. 325-349, Birkhäuser/Springer Basel AG, Basel, 2012, doi:10.1007/978-3-0348-0411-0_23.

[34] D. G. Wagner, Multivariate stable polynomials: theory and applications, Bull. Amer. Math. Soc. (N.S.) 48 (2011), no. 1, 53-84, doi:10.1090/S0273-0979-2010-01321-5.

Department of Mathematics

Washington University in St. Louis

St. Louis, MO 63130

USA

geknese@wustl.edu 